\title{
Internal current measurements in high power impulse magnetron sputtering
}

Daniel Lundin, Seham Al Sahab, Nils Brenning, Chunqing Huo and Ulf Helmersson

\section{Linköping University Post Print}

N.B.: When citing this work, cite the original article.

Original Publication:

Daniel Lundin, Seham Al Sahab, Nils Brenning, Chunqing Huo and Ulf Helmersson, Internal current measurements in high power impulse magnetron sputtering, 2011, Plasma sources science \& technology (Print), (20), 4, 045003.

http://dx.doi.org/10.1088/0963-0252/20/4/045003

Copyright: Institute of Physics

http://www.iop.org/

Postprint available at: Linköping University Electronic Press

http://urn.kb.se/resolve?urn=urn:nbn:se:liu:diva-56743 


\title{
Internal current measurements in high power impulse magnetron sputtering
}

\author{
Daniel Lundin ${ }^{1}$, Seham Al Sahab ${ }^{1}$, Nils Brenning ${ }^{2}$, Chunqing Huo ${ }^{2}$, and Ulf \\ Helmersson ${ }^{1}$
}

${ }^{1}$ Plasma \& Coatings Physics Division, IFM-Materials Physics, Linköping University, SE-581 83 Linköping, Sweden

${ }^{2}$ Division of Space and Plasma Physics, School of Electrical Engineering, Royal Institute of Technology, SE-100 44 Stockholm, Sweden

E-mail: daniel.lundin@liu.se

\begin{abstract}
The transport of charged particles in a high power impulse magnetron sputtering (HiPIMS) discharge is of great interest when optimizing this promising deposition technique with respect to deposition rate and control of the ion acceleration. In the present study the internal current densities $J_{\varphi}$ (azimuthal direction) and $J_{z}$ (axial direction) have therefore been spatially and temporally resolved in the bulk plasma region above a cylindrical magnetron using Rogowski coils. From the measurements a phenomenological model has been constructed describing the evolution of the current density in this pulsed plasma. The core of the model is based on three different types of current systems, which characterize the operating transport mechanisms, such as
\end{abstract}


current transport along and across magnetic field lines. There is a gradual change between these current systems during the initiation, build-up and steady-state of a HiPIMS plasma. Furthermore, the data also shows that there are spatial and temporal variations of the key transport parameter $J_{\varphi} / J_{z}$, governing the cross-B resistivity and also the energy of the charged particles. The previously reported faster-than-Bohm cross-B electron transport is here verified, but not for all locations. These results on the plasma dynamics are essential input when modeling the axial electric field, governing the back-attraction of ionized sputtered material, and might furthermore provide a link between the different resistivities reported in HiPIMS, pulsed-DC, and DC magnetron discharges. 


\section{Introduction}

High power impulse magnetron sputtering (HiPIMS) introduced by Kouznetsov et al. in 1999 [1] is a promising technique for improving magnetron sputtering used in many industrial processes for thin film deposition [2]. The HiPIMS discharge generates a highly ionized plasma with large quantities of energetic metal ions [3] due to very high pulse power densities with, in some cases, a directed flux of charged species [4]. The high degree of ionization of the sputtered species has several advantages for thin film growth, such as an increased density [5] and adhesion [6], in particular for complex-shaped surfaces [7, 8], and a decreased deposition temperature [9].

The high potential applied to the magnetron in a HiPIMS discharge generates large internal currents in the plasma between the sputtering cathode and the anode (often the chamber walls and the ground shield of the magnetron). Large azimuthal currents, circulating in the magnetic field trap close to the target surface, also arise due to the presence of the magnetic field [10]. Measurements by Bohlmark et al. [11] show that the ratio between the azimuthal Hall current density, $J_{H}$, and the cross-B discharge current density, $J_{D \perp}$, in a typical HiPIMS process is surprisingly low, meaning a high speed in the electron transport across the magnetic field lines during the HiPIMS discharge. This so-called anomalous transport is known to also affect the transport of ions in this type of discharge [4] and has recently been investigated in more detail using electric probes [10] to estimate the azimuthal current, $J_{\varphi}$. From these studies it was concluded that the current density ratio was typically $J_{\varphi} / J_{D \perp} \approx 2$. This value is significantly lower than what is expected from classical theory of diffusion and 
electrical conductivity using collisions to move electrons across the magnetic field, or even Bohm diffusion seen in common direct current magnetron sputtering (DCMS) [10]. Expected values for the Bohm regime is in the range $8<J_{\varphi} / J_{D \perp}<30[12,13]$. Moreover, in a recent series of experiments and simulations [10, 14] it was proposed that the anomalous transport could be described as being mediated by highly nonlinear waves, likely due to the modified two-stream instability (MTSI), resulting in electric field oscillations in the MHz-range. Furthermore, the ratio $J_{\varphi} / J_{D \perp}$ can in the magnetron geometry be identified with the more general Hall parameter $\omega_{g e} \tau_{E F F}$ [10]. Here $\omega_{g e}$ is the electron (angular) gyrofrequency and $\tau_{E F F}$ is the effective electron-ion momentum transfer time. In a study by Brenning et al. [15] analyzing three different types of pulsed plasma discharges (including HiPIMS) of different magnetic geometry, as well as covering several orders of magnitude in plasma density, it was found that the faster-than-Bohm anomalous transport is present in all cases and is expressed by $\omega_{g e} \tau_{E F F}=2.7$ within a factor of 2 . This gives an approximate fasterthan-Bohm transport range of $1.5<\omega_{g e} \tau_{E F F}<5.5$.

In summary, previous investigations have provided important insights into understanding the mechanism behind the anomalous transport, but still it is likely that the same pulsed discharge can undergo different transport types in different parts of the plasma as well as during different stages of the pulse. In order to fully understand the transport of the energetic charged particles it is necessary to both spatially and temporally resolve what type of transport mechanism that operates in the HiPIMS discharge. In this study the current densities $J_{\varphi}$ and $J_{z}$ have therefore been directly measured using Rogowski coils, which, unlike electrical probes, require no further 
information regarding the plasma characteristics in order to determine the current flowing in a particular direction. The current density ratio, $J_{\varphi} / J_{z}$, has also been calculated and is here presented for different locations in the plasma as well as for different times during the pulse. Above the racetrack the magnetic field is approximately parallel to the target, $J_{z} \approx J_{D \perp}$, and the measured current density ratio gives the Hall parameter: $J_{\varphi} / J_{z} \approx J_{\varphi} / J_{D \perp}=\omega_{g e} \tau_{E F F}$.

\section{Experimental details}

The experiments were carried out using a standard, circular, slightly unbalanced, planar magnetron source equipped with a high-purity copper $(\mathrm{Cu})$ target with a diameter of $0.150 \mathrm{~m}$. The magnetic null point is located about $0.10 \mathrm{~m}$ from the target surface and the target race track is approximately located at $\rho=4.5 \mathrm{~cm}$. A complete description of the magnetic field topology of the present magnetron setup has been given by Bohlmark et al. [11]. The magnetron (cathode) was located inside a stainless steel vacuum chamber (height $0.70 \mathrm{~m}$, diameter $0.44 \mathrm{~m}$ ). The chamber walls and the ground shield of the magnetron served as an anode for the discharge. The base pressure was maintained around $1.33 \times 10^{-4} \mathrm{~Pa}$ using a turbo molecular-pump. Argon with a minimum purity of $99.9997 \%$ was used as a discharge gas and introduced into the chamber through a mass flow controller. The argon pressure was kept constant at $0.53 \mathrm{~Pa}$.

Unipolar pulses were applied between the cathode (target) and the chamber walls using a HiPIMS Sinex 3 (Chemfilt Ionsputtering AB, Sweden) power supply capable of delivering $2000 \mathrm{~V}$ and $1200 \mathrm{~A}$ peak values. In this work pulses up to approximately 
$800 \mathrm{~V}$ and $145 \mathrm{~A}$ were used with a pulse duration of $200 \mu$ s and a repetition frequency of $100 \mathrm{~Hz}$, as seen in Figure 1.

In this work Rogowski coils [16] were used for measuring the current density. The probe consists of a helical coil of wire with the lead from one end returning through the centre of the coil to the other end, so that both terminals are at the same end of the coil in order to eliminate contributions from other components of the current. The Rogowski coil has no ferromagnetic material in the core, which means that the coil can not be driven into saturation and therefore responds linearly to extremely large currents [17]. Being of low inductance it can also respond to very high frequency pulses, and thus making it suitable as a plasma diagnostics tool in HiPIMS discharges. The probe was mounted on a movable metal rod for scans taken at different axial $(z)$ as well as radial $(\rho)$ positions. In this work $z=0$ marks the target surface and $\rho$ is measured from the center axis of the target. The center of the probe torus was chosen to define the probe position. The probe was never moved closer than $z=4 \mathrm{~cm}$ in order not to disturb the plasma discharge. Furthermore, the probe and rod could be rotated in order to measure directional currents. In the present investigation the axial as well as azimuthal current profiles were determined in the region $4 \leq z \leq 8 \mathrm{~cm}$ and $0 \leq \rho \leq 7.5 \mathrm{~cm}$ assuming axial symmetry. Data was collected at $1 \mathrm{~cm}$ intervals in the axial direction and at four different points in the radial direction $(\rho=0,2.5,4.5,7.5$ $\mathrm{cm})$, constituting a total of 20 different locations in the plasma.

A Rogowski coil produces a voltage signal [16] given as

$$
V_{\text {coil }}=\frac{n A \mu_{0}}{2 \pi r} \frac{d I}{d t},
$$


where $n$ is the number of turns, $A$ is the cross-sectional area for each turn, $r$ is the major radius of the coil torus, and $I$ is the current through it. The probe signal is integrated to yield the current as function of time. It is not possible to measure DC currents with this type of probe, since the voltage that is induced in the coil is proportional to the rate of change of the current.

In the present experiments a Rogowski coil, where the shield had inner radius of 9.7 $\mathrm{mm}$ and outer radius of $21.5 \mathrm{~mm}$, was inserted into the plasma. The current density was obtained from the measured total current by assuming an effective collection radius of $15.6 \mathrm{~mm}$, with an estimated error of $50 \%$ due to the uncertainty of how the current pattern is influenced by the probe: deflected around the probe torus, or compressed into it. The winding consisted of $0.2 \mathrm{~mm}$ copper wire around a rigid plastic toroidal core making up a total of 470 turns. The probe was calibrated several times throughout the whole series of measurements by recording a known sinusoidal current running through the center of the probe together with the probe output. The probe signal was later on integrated and manually fitted to the current signal. From the calibration it was concluded that the probe provided very good accuracy up to $1 \mathrm{MHz}$, and thus meeting the experimental requirements. Furthermore, careful precautions were taken to minimize the system noise, which meant adding additional shielding to cables from the turbo pump as well as control units. In this way the maximum noise level was found to be less than $5 \%$ by measuring the probe signal inside the chamber with no process running as well as during operation. Basic plasma parameters such as target voltage and target current as well as the probe signal were monitored and recorded on a Tektronix TDS2004B oscilloscope. A Tektronix P6015 high voltage probe measured the target voltage. The current was measured with a Chauvin Arnoux 
C160 current probe. All signals recorded were averaged over 128 measurements to ensure better statistics.

\section{Results and discussion}

\subsection{Internal current distributions}

The internal currents $J_{\varphi}$ and $J_{z}$ in a typical HiPIMS discharge were measured in the ranges $4 \leq z \leq 8 \mathrm{~cm}$ and $0 \leq \rho \leq 7.5 \mathrm{~cm}$. An example of a time-resolved current density curve of the $J_{\varphi}$ component taken at $z=7 \mathrm{~cm}$ and $\rho=4.5 \mathrm{~cm}$ is given in Figure 2. The letters a-e indicate the different times where current density maps have been generated. The most striking features are the two peaks present at about $60 \mu \mathrm{s}$ and $130 \mu \mathrm{s}$. They were seen in all measurements, although the magnitude of the individual peaks as well as the relative magnitude between the two peaks shifted depending on location. The time of the first peak always coincides with the peak of the discharge current for both $J_{\varphi}$ and $J_{z}$. Table 1 summarizes the different features for the chosen points in time.

\begin{tabular}{cc|c}
\hline Point & $\begin{array}{c}\text { Time after pulse } \\
\text { initiation }[\mu \mathrm{s}]\end{array}$ & Feature \\
\hline a & 30 & Early stage of the discharge pulse $\left(I_{D} \sim 60 \mathrm{~A}\right)$ \\
b & 60 & Peak of discharge current $\left(I_{D} \sim 145 \mathrm{~A}\right)$, first peak in the \\
& & Dinternal current density \\
c & 85 & decreasing $\left(I_{D} \sim 115 \mathrm{~A}\right)$ \\
$\mathrm{d}$ & 100 & New increase of internal current density, decreasing \\
\hline
\end{tabular}


discharge current $\left(I_{D} \sim 90 \mathrm{~A}\right)$

Second peak in the internal current density, knee in the

e

130

discharge current $\left(I_{D} \sim 60 \mathrm{~A}\right)$

Table 1. A summary of the features seen in the discharge current as well as the general trends for a typical internal current density measurement for five different stages of the HiPIMS pulse. The points a-e is below plotted as current density maps for the investigated region (see Figure 3a-e).

In order to facilitate the interpretation of the evolving internal currents present during the HiPIMS discharge, 2D current density maps have been generated based on the results from the measurements. These maps are given in Figure 3a-e, where the letter stands for the same point in time as seen in Table 1. The left panel is the measured $J_{\varphi}$, the middle panel represents $J_{z}$, and the right panel is the current density ratio $J_{\varphi} / J_{z}$. Since the color coding varies between these current density plots, they have for clarity been provided with a scale factor in the upper left corner indicating the approximate current density increase compared to Figure 3a. Given below is first a description of the currents measured during the HiPIMS discharge, which is followed by an analysis of the internal current system.

Following the time-evolution of the internal currents it is seen in the middle panel of Figure 3 a that only small axial currents are flowing above $z=4 \mathrm{~cm}$ during the initial part of the HiPIMS discharge $(t<30 \mu \mathrm{s})$. From the $J_{z}$ plot at $30 \mu \mathrm{s}$ it can be concluded that most of the current is transported from the center and outer edges of the magnetron to the nearest anode point along the magnetic field lines and that little axial cross-B transport is present above the race track region. At the same time a clearly distinguished azimuthal current is flowing in a torus $5 \mathrm{~cm}$ above the target race 
track. This is in line with electron density measurements by Bohlmark et al. [18] on the same system, where they recorded a toroidal-shaped density increase at the same time and place. Also, in a previous study on Hall currents using the same setup (but a Ta target) it was measured that, in the early phase of the discharge (at $16 \mu \mathrm{s}$ ), $J_{\varphi}$ reached values around $10-20 \mathrm{kA} \mathrm{m}^{-2}$ at $4-6 \mathrm{~cm}$ above the target race track for 5-6 times the discharge current ( 300-350 A) compared to the present investigation (60 A) [11]. The left panel of Figure 3a shows slightly less than a quarter of that value, which is consistent with our lower discharge current. Moreover, it is seen that further away from the cathode surface $(z>6 \mathrm{~cm})$ both $J_{\varphi}$ and $J_{z}$ are of the same magnitude, indicating that here is a region of much larger cross-B transport (i.e. lower Hall parameter $\left.\omega_{g e} \tau_{E F F}\right)$.

At the peak of the discharge current $(t=60 \mu \mathrm{s})$ the azimuthal current is fully developed and reaches values around $22 \mathrm{kA} \mathrm{m}^{-2}$ above the target race track (Figure $3 \mathrm{~b}$ ), which is a tenfold increase compared to $t=30 \mu \mathrm{s} . J_{z}$ has now also developed in the region above the target race track, although it only amounts to about twice the increase in magnitude in comparison with $t=30 \mu \mathrm{s}$. If assuming that most of the current at this stage in the pulse runs in the azimuthal direction in a plasma torus (inner radius $3.5 \mathrm{~cm}$ and outer radius $7.5 \mathrm{~cm}$ ) with its center located just below $z=4$ $\mathrm{cm}$ and with the above given current density, then the total azimuthal current is approximately $28 \mathrm{~A}$, which is roughly $20 \%$ of the discharge current.

After $t=85 \mu \mathrm{s} J_{z}$ is found to increase faster than $J_{\varphi}$, as seen in Figure 3c-d, meaning that there is a comparably lower Hall parameter $\omega_{g e} \tau_{E F F}$ in this time interval, which 
will be further analyzed in section 3.3. $J_{\varphi}$ still preserves the characteristic toroidal current flow, which is further expanded in space as the internal current densities reach a second maximum at $t=130 \mu$ s seen in Figure 3e. $J_{\varphi}$ above the race track at $z \approx 4$ $\mathrm{cm}$ is found to be more than ten times larger than $J_{z}$.

In Figure 3a-e of $J_{z}$ two current-intense areas can be identified: 1) At $z \sim 4 \mathrm{~cm}$ and $\rho$ $\sim 0 \mathrm{~cm}$ as well as 2) at $z \sim 4 \mathrm{~cm}$ and $\rho \sim 7.5 \mathrm{~cm}$. These must be mainly field-aligned currents $J_{\|}$, since the magnetic field lines at these locations run almost perpendicular to the magnetron surface, as can be seen in Figure 4. In order to study the edge effects of the cross-B electron transport it is therefore necessary to investigate the radial component of the current density distribution. Only above the target race track, where a large part of the cross-B current is carried, $J_{\varphi} / J_{z} \approx \omega_{g e} \tau_{E F F}$ is valid, and will be used in the analysis below.

Another way of illustrating the measured current density dynamics from ignition, via peak to decay of the HiPIMS discharge, is to look at the net current flow across the different boundaries of the investigated cylindrical volume $(4 \leq z \leq 8 \mathrm{~cm}$ and $0 \leq \rho \leq 7.5 \mathrm{~cm})$. These currents have been calculated from the data in Figure 3a-c using Kirchhoff's current law and are given in Figure 4 in percent of the discharge current. The block arrows show the total current $I$ across the indicated surface (i.e. not the current density $J$ ). A few things can be noted: (1) During the rise of the pulse, most of the discharge current crosses the B-field radially and close to the target surface $(z<4 \mathrm{~cm})$. As the pulse evolves, this fraction decreases dramatically (only $12 \%$ at $t=85 \mu \mathrm{s})$. (2) Cross-B current transport across the lower disc $(z=4 \mathrm{~cm}$, 
$0 \leq \rho \leq 7.5 \mathrm{~cm}$ ) is not detected during the rise of the pulse but grows to be the dominant fraction of the measured total current as the discharge decay phase is reached ( $88 \%$ at $t=85 \mu \mathrm{s})$. (3) There is a radial current reversal between 60 and 85 $\mu$ s across the cylinder surface $(4 \leq z \leq 8 \mathrm{~cm}, \rho=7.5 \mathrm{~cm})$, which is analyzed in more detail in the next section.

\subsection{Analysis of the current system in the bulk plasma}

Analysis of the data in Figure 3a-c shows that the internal currents in the volume of the discharge goes through a sequence of configurations during the pulse. We propose that this complex time evolution is suitably analyzed in three types of current systems,

called I, II (subdivided into IIa, IIb, IIc), and III that are shown in Figure 5 and summarized in Table 2. It will be shown that a suitable superposition of these current systems can describe the internal current dynamics for any given time during the HiPIMS discharge.

\begin{tabular}{|c|c|}
\hline $\begin{array}{l}\text { Current } \\
\text { configuration }\end{array}$ & Feature \\
\hline $\begin{array}{l}\text { I } \\
\text { dominates } \\
\text { during } \\
\text { ignition }\end{array}$ & $\begin{array}{l}\text { Ionization close to the target surface, close to the anode ring. Too } \\
\text { low plasma density in the bulk volume (away from the target) for } \\
\text { electron transport through this region. Radial cross-B transport close } \\
\text { to the target. }\end{array}$ \\
\hline $\begin{array}{l}\text { IIa to IIc } \\
\text { transition }\end{array}$ & $\begin{array}{l}\text { Ionization close to the target surface, but away from the race track. } \\
\text { Sufficient plasma density in the bulk plasma, so that electrons can }\end{array}$ \\
\hline
\end{tabular}


move through this region: first along the magnetic field lines and then, in the weaker $\mathbf{B}$ at high $z$, across.

III Ionization above the target race track. Electrons have to go across $\mathbf{B}$ dominates in steady state (and DCMS)

the whole way through the bulk plasma, until they reach the first grounded magnetic field line.

Table 2. A proposed classification of the current systems operating during the HiPIMS discharge.

We propose that that the redistribution of currents observed in Figure 4 can be understood in terms of the current systems of Figure 5 as follows:

(1) Each current pulse starts with an ignition-phase during which there is negligible plasma in the bulk volume of the vessel. Since the vacuum electric field is strongest in the vicinity of the anode ring of the magnetron, this is a natural place of ignition, beginning as a localized glow discharge close to the target surface. Without plasma in the bulk volume, the electrons can in this ignition phase only go in a current system of type I to the anode ring. This is why there is a strong radial current close to the target surface during the rise of the HiPIMS pulse, as seen in Figure 4a.

(2) The next phase is the bulk plasma breakdown. Secondary electrons, and electrons created in the ionization region close to the target, are accelerated out along the magnetic field into the bulk volume and begin to ionize the neutral gas. When the bulk plasma density has built up sufficiently to admit current closure across $\mathbf{B}$ at high $z$, a current system of types IIa-IIc is added to the overall current distribution (Figure 4b). At $30 \mu$ s, type IIa carries $20 \%$ of the discharge current (see Figure 4a). In the 
current system types IIa and IIb, the transport of electrons along B should be associated with two $J_{z}$ current maxima at $\mathrm{z} \approx 4 \mathrm{~cm}$ : one around $\rho=0 \mathrm{~cm}$ and another around $\rho \approx 8 \mathrm{~cm}$, where the magnetic field lines are aligned in the $z$ direction. Both $J_{z}$ maxima are clearly present in the experimental data (middle panel of Figure 3a).

(3) The final phase is establishing steady state. As can be seen in the middle panels of Figure 3a-e there is a transition in $J_{z}$ from the above described maxima at the center and edge of the target (types IIa and IIb in Figure 5b) to a more evenly distributed axial current density ( $\mathrm{t} \geq 85 \mu \mathrm{s})$, indicating an increased cross-B transport above the race track (type IIc and III). Eventually, approaching the steady state, a type III current system is dominating. At this stage the plasma density above the race track is high enough that this route is the easiest for the electron current to cross the magnetic field lines. The gradual change of current systems has been estimated from the data in Figure 4 and is summarized in Figure 6, from which it can be concluded that steady state is approached somewhere around $85-100 \mu$ s. Due to the short pulse length in this experiment no real steady state situation is established, since the discharge goes into the decay phase.

An unexplained feature during the decay phase is the second axial as well as azimuthal current peak seen for the azimuthal case in Figure $2(t=130 \mu \mathrm{s})$. At this point the axial current density is still high (middle panel of Figure 3e), which is out of phase with the decreasing discharge current $\left(I_{D} \sim 60 \mathrm{~A}\right)$ and clearly very different to the ignition phase current density distribution seen in the middle panel of Figure 3a. It might be that both the azimuthal and the cross-B current are increasingly concentrated in the region above the race track because the plasma density still rises there. Similar 
double peak structures were observed by Bohlmark et al. [11] when investigating time-dependent variations of the magnetic field on the same device. The authors ascribe this phenomenon as related to an interplay between the electric field (-z) and the plasma pressure $(+z)$, where the electric field is strongly reduced in the decay phase of the HiPIMS pulse, and thereby causing an expansion of the dense plasma torus.

\subsection{Current ratio}

The spatially resolved current density ratio $J_{\varphi} / J_{z}$ is shown in the right hand panels of Figure 3a-e. The reason for investigating this ratio is the previously discussed anomalous cross-B transport of electrons, which has been described in several earlier works $[4,10,12,14,15]$. A short summary of this particular transport mechanism is given below to facilitate the discussion of the results that follow.

In the HiPIMS plasma the classical resistivity, governing the transport properties, is modified by the existence of a plasma instability [10], driven by the azimuthal current. The correlation between oscillating $n_{e}$ and $E_{\varphi}$ results in azimuthal forces of opposite sign on the electron and ion populations. Macroscopically, this is equivalent to an anomalous cross-B resistivity, $\eta_{\perp}=<n_{e} E_{\varphi}>/\left(J_{\varphi}<n_{e}>\right)$ [4]. The effective electron momentum transfer time, often called the effective electron collision time, $\tau_{E F F}$, is related to the cross-B resistivity through

$$
\eta_{\perp}=\frac{m_{e}}{\tau_{E F F} e^{2} n_{e}}=\frac{B}{\omega_{g e} \tau_{E F F} e n_{e}}
$$


where the electron angular gyro frequency is written as $\omega_{g e}=e B / m_{e}[19]$. In the magnetron geometry, the product $\omega_{g e} \tau_{E F F}$ can be found from a macroscopic measurement of the current density ratio $J_{\varphi} / J_{D \perp}=\omega_{g e} \tau_{E F F}$ (where it is assumed that $J_{D \perp}$ is carried by electrons) $[12,14]$. This means that a low ratio of $J_{\varphi} / J_{D \perp}$ (or equivalently a low $\omega_{g e} \tau_{E F F}$ ) is the result of increased transverse resistivity and thereby increased cross-B conductivity and diffusion of electrons. As mentioned in the introduction, earlier estimates of $J_{\varphi} / J_{D \perp}$ in magnetrons show values that are around 2 in HiPIMS [10, 11] and in the range $8-30$ in DCMS discharges [12, 13]. The estimates in HiPIMS magnetrons are, however, based on assumptions about the spatial distribution of $J_{z}$ over magnetic flux surfaces, and furthermore are time averages around the time of the discharge current maximum. Through the use of the Rogowski probe these limitations have been overcome and the first time and space resolved current density ratios are here presented. As described above, the current density measurements cover only $J_{\varphi}$ and $J_{z}$ (not $J_{r}$ ), and $\omega_{g e} \tau_{E F F}$ can only be obtained above the race track region, where the magnetic field is parallel to the target surface.

Figure 7 shows $J_{\varphi} / J_{z}$ above the race track for times 30, 60, 85, 100, and $130 \mu \mathrm{s}$ based on the results in the right panels of Figure 3a-e. The following observations can be made: (1) There are small variations in time, where the values stay within a factor of two from the average indicated by a solid line. The lowest $\omega_{g e} \tau_{E F F}$ values are found at the times of the transition regions of the internal current measurements, i.e. point a, c and d in Figure $2(t=30,85$ and $100 \mu$ s respectively). (2) There is a systematic 
spatial variation. Only around $z \sim 7-8 \mathrm{~cm}$ are the earlier reported low values $\omega_{g e} \tau_{E F F} \approx 2$ recovered. Closer to the target, $\omega_{g e} \tau_{E F F}$ increases with decreasing $z$ and values approaching the Bohm range are found. (3) A comparison with the magnetic field strength $B(z)$ shows that $\omega_{g e} \tau_{E F F}$ scales approximately as $1 / B$, as expected from classical collisions with constant $\tau_{E F F}$. However, classical collisions (also plotted for reference in Figure 7) are over the whole range of $z$ and at all times about a constant factor of 8 too infrequent. Further analysis of this topic is postponed until we have a firmer experimental basis, including measurements of the $J_{r}$ component.

\section{Conclusions}

The internal current densities $J_{\varphi}$ and $J_{z}$ have been successfully measured in a HiPIMS discharge using Rogowski coils. From the spatially and temporally resolved data it is seen that the current density evolution can be characterized by a gradually changing superposition of three different types of current systems operating during the pulse: type I, IIa-c and type III, with which the development of the pulsed plasma can be described in a simple phenomenological model. Also the mechanisms of current transport along, as well as across magnetic field lines are covered in the present description. Furthermore, the current density ratio, $J_{\varphi} / J_{D}$, has also been calculated due to its importance for characterizing the cross-B electron transport in these types of discharges. It was found that faster-than-Bohm cross-B electron transport is operating in the HiPIMS discharge, in particular during the internal current transient regimes for $z>6 \mathrm{~cm}$, whereas Bohm diffusion is approached for $\mathrm{z}<5 \mathrm{~cm}$. 


\section{Acknowledgements}

The authors gratefully acknowledge Michael Raadu for valuable discussions, and

Petter Larsson for technical assistance in the manufacturing of the Rogowski probes.

This work was partly supported by the Swedish Research Council and the Swedish Foundation for Strategic Research. 


\section{Figures}

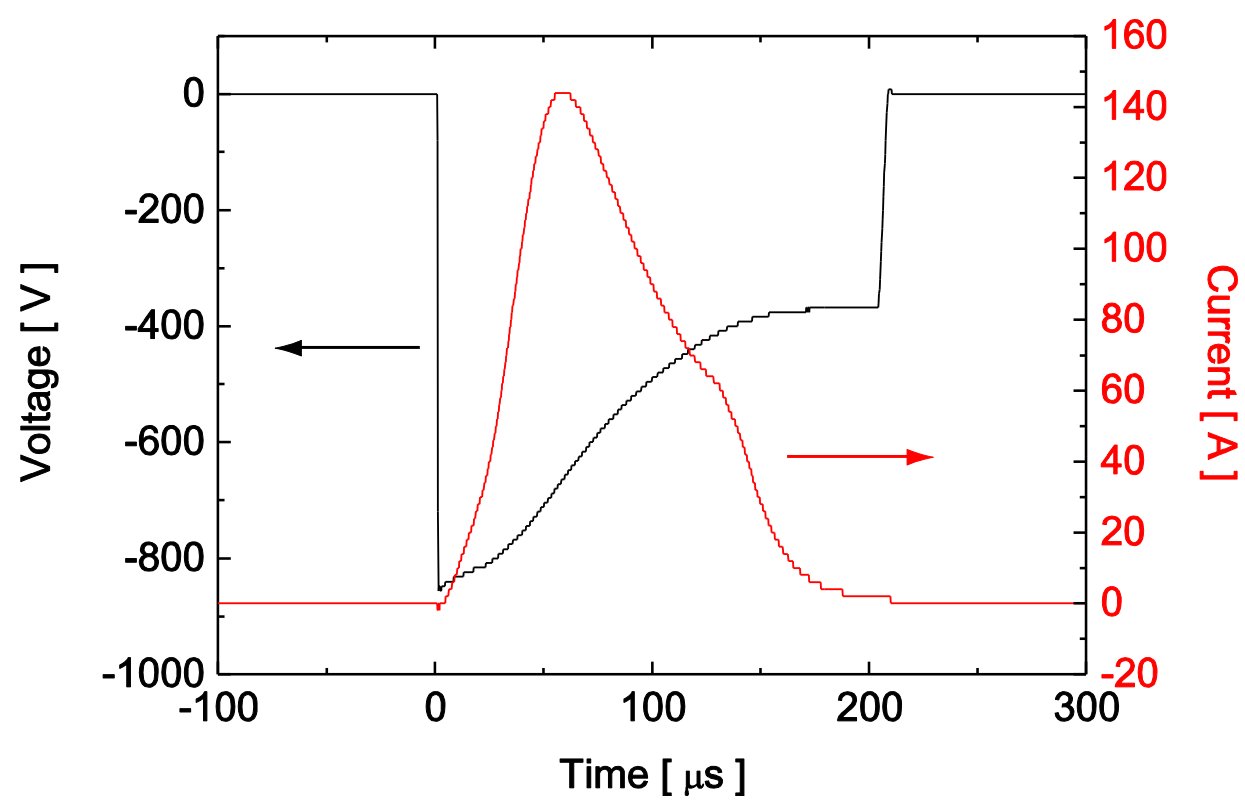

Figure 1. Voltage and current characteristic for a typical HiPIMS discharge pulse used in the study.

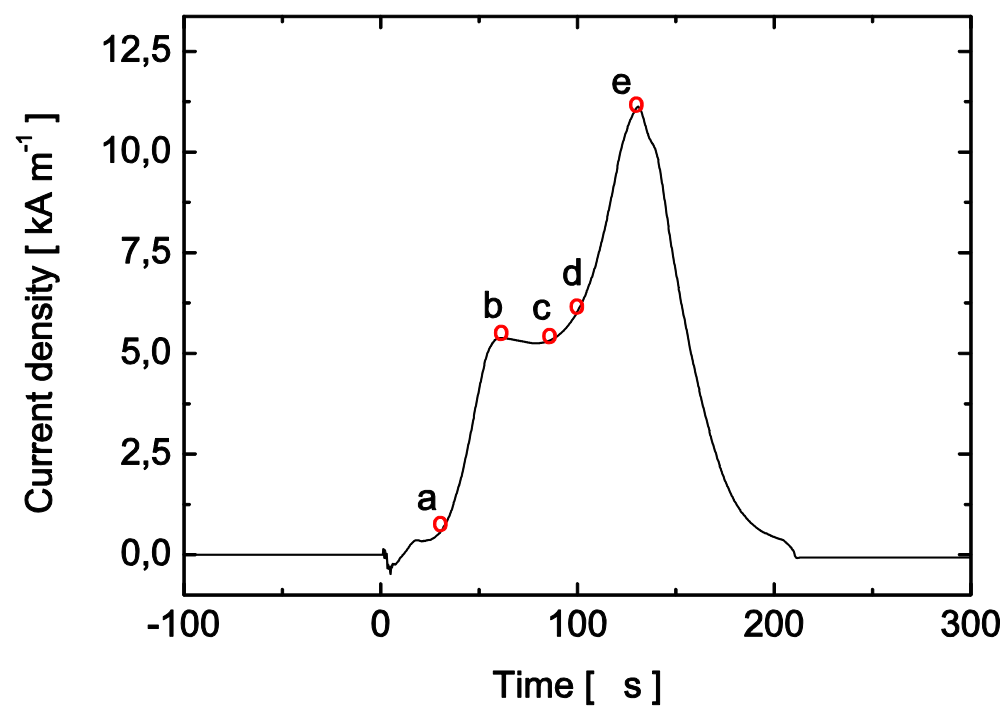

Figure 2. A typical internal current density measurement generated by integrating the signal from the Rogowski coil. The letters a-e indicate the different times where current density maps have been created. This particular profile was recorded when measuring $J_{\varphi}$ at $z=7 \mathrm{~cm}$ and $\rho=$ $4.5 \mathrm{~cm}$ (i.e. above the target race track). 
$J_{\varphi}$

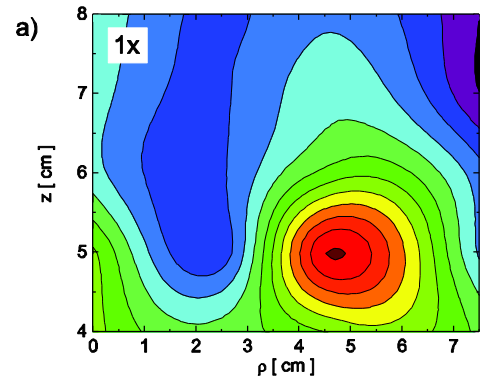

b)

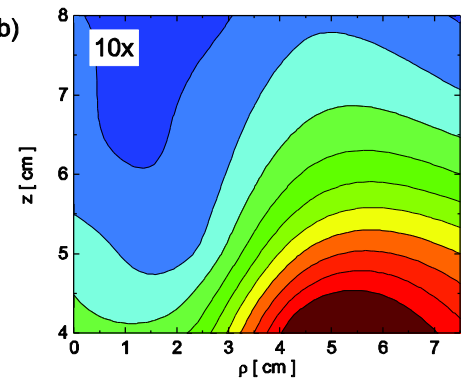

c)

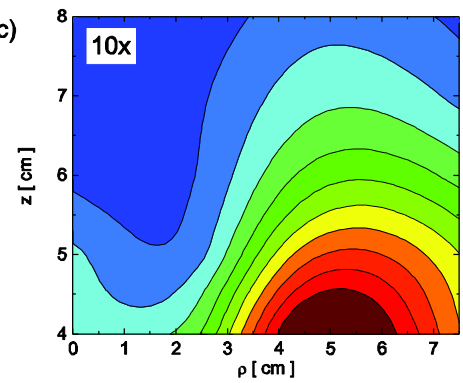

d)

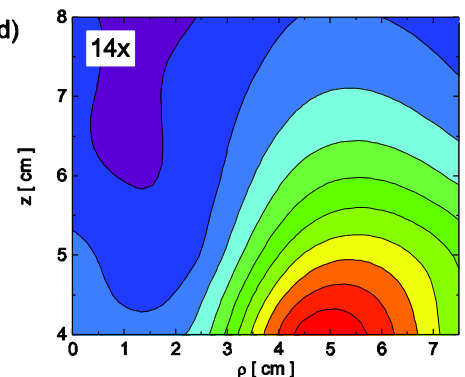

e)
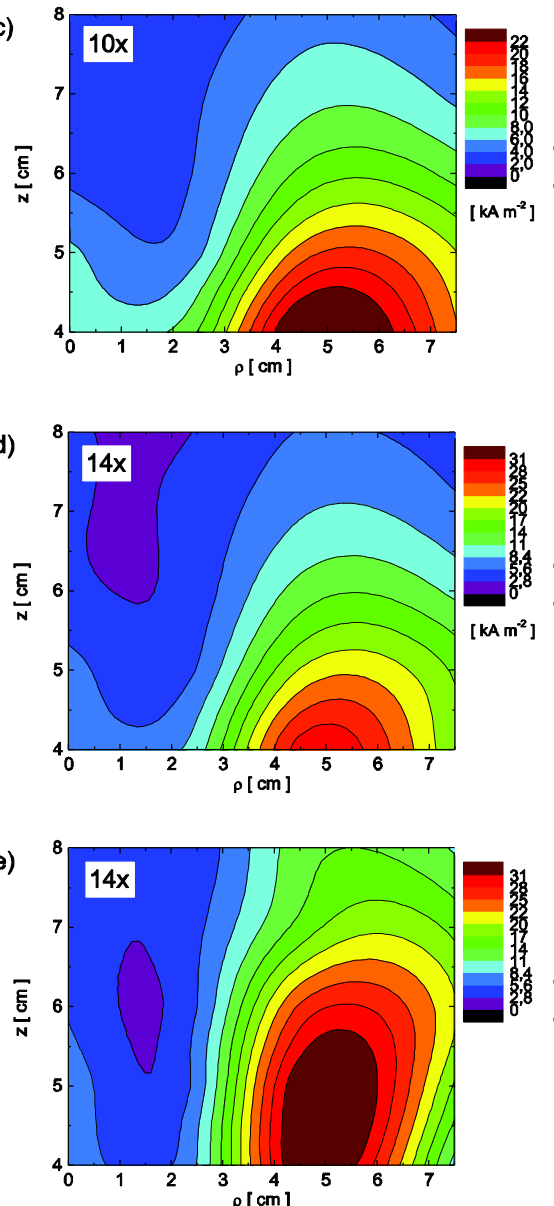
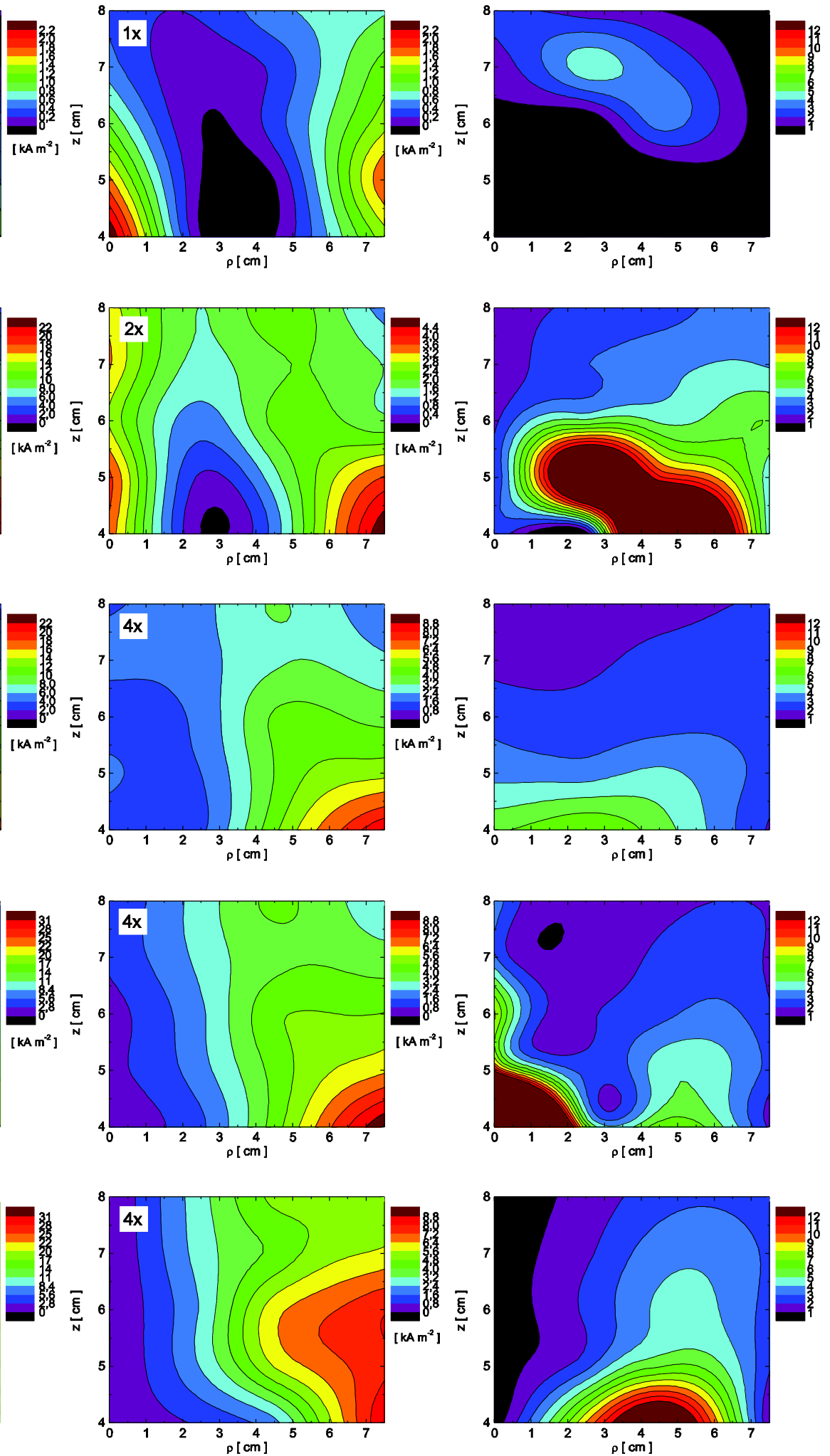

Figure 3. Current density maps of $J_{\varphi}$ (left) and $J_{z}$ (middle) as well as the current density ratio $J_{\varphi} / J_{z}$ (right) taken at a) $30 \mu \mathrm{s}$, b) $60 \mu \mathrm{s}$ c) $85 \mu \mathrm{s}$ d) $100 \mu \mathrm{s}$ and e) $130 \mu \mathrm{s}$ after pulse initiation.

Notice that the current density values in Figure 3b-e are higher compared to Figure 3a, which is 
represented by an approximate scale factor in the upper left corner. The hatched area in the right panel of Figure 3a indicates that no current ratio could be established here due to a zero $J_{z}$ value. 

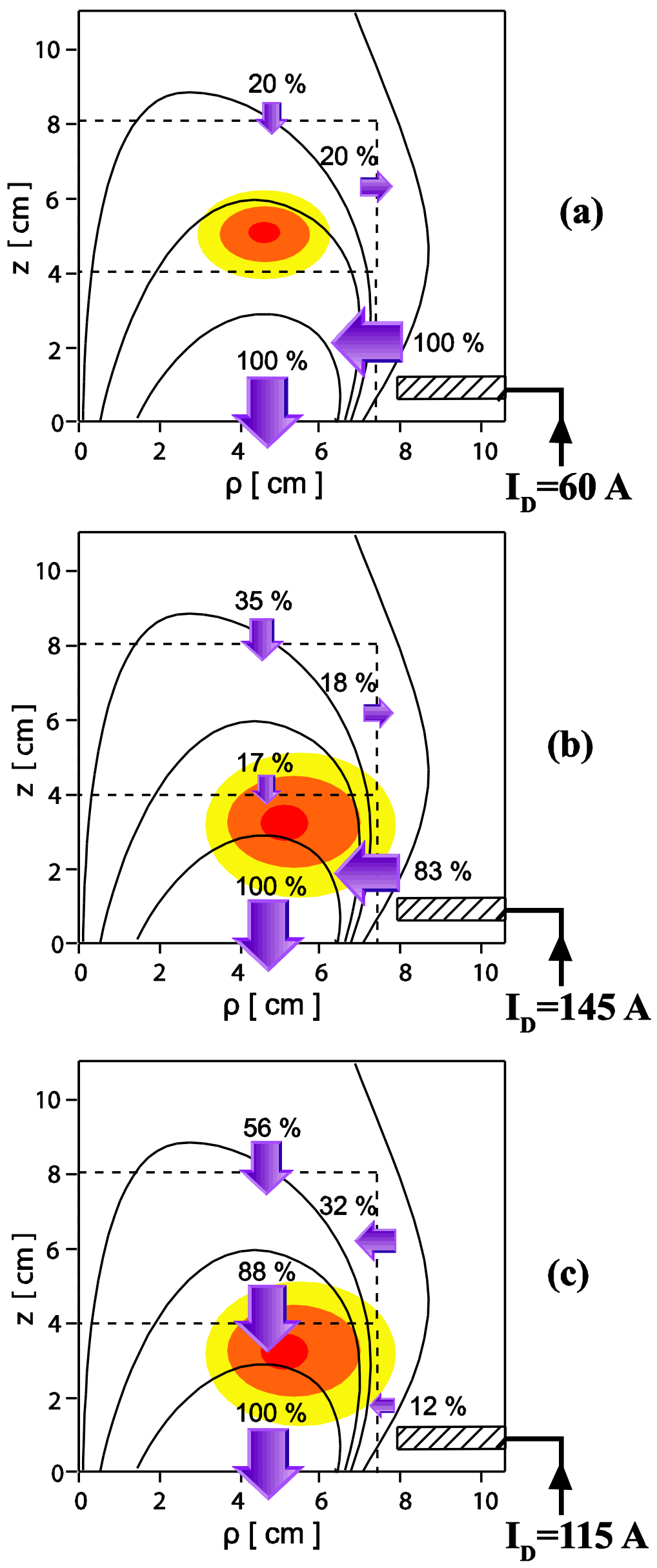
Figure 4. Analysis of the measured current densities from Figure 3 in terms of how the current is transported across the investigated region. (a) At $t=30 \mu \mathrm{s}$, during the breakdown phase, (b) at $t$ $=60 \mu$ s during the maximum of the discharge current and (c) at $t=85 \mu$ s during the decay of the HiPIMS discharge current. The net current flow across the different boundaries of two cylindrical volumes in the discharge, between $z$ coordinates $0-4 \mathrm{~cm}$ and $4-8 \mathrm{~cm}$ respectively, is shown. The areas of the block arrows are proportional to the currents, which are also given in percent of the discharge current. The ellipses above the target race track represent cross sections of the dense plasma torus also seen in the $J_{\varphi}$ plots of Figure 3 (left panels). 

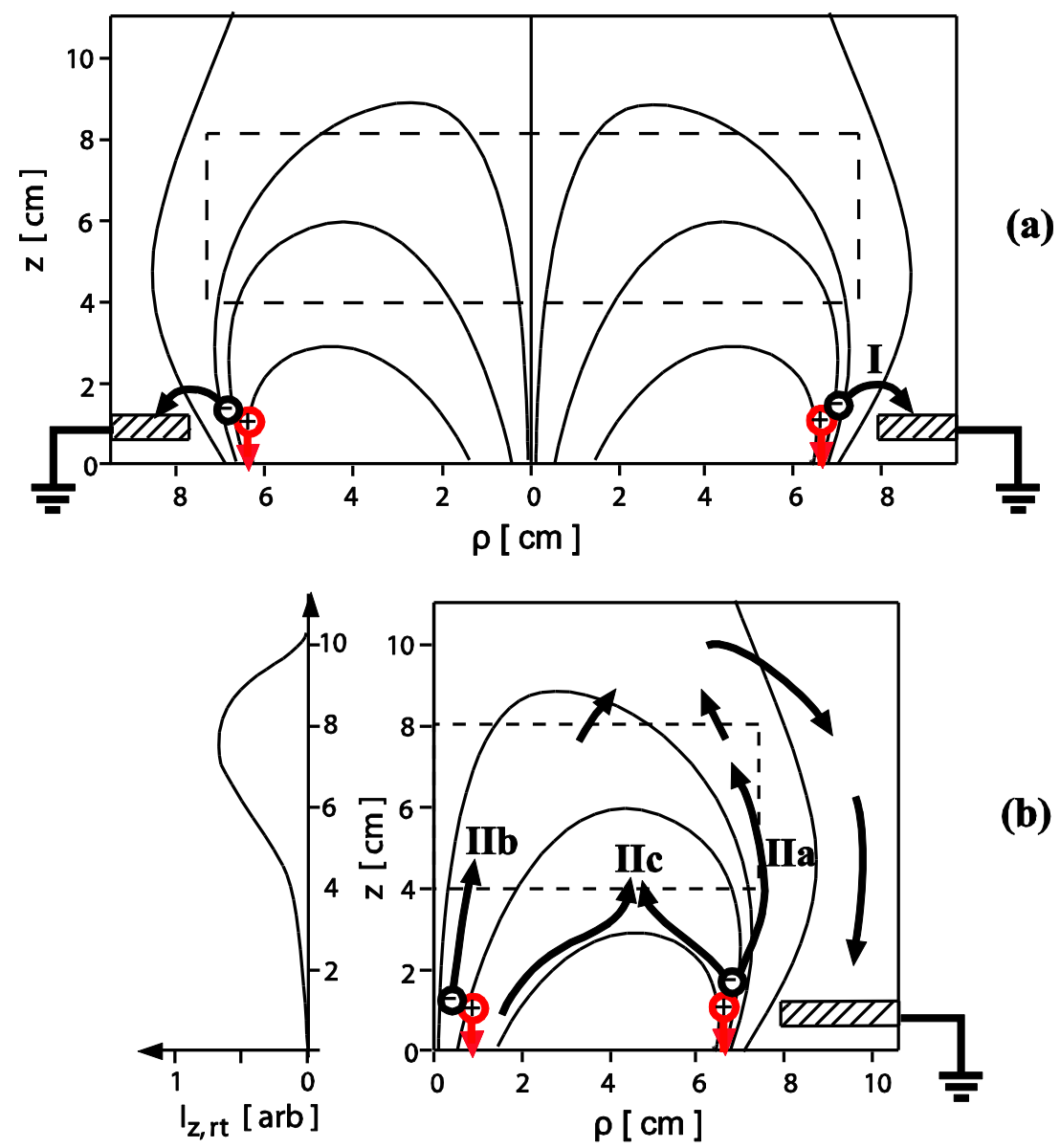

(b)

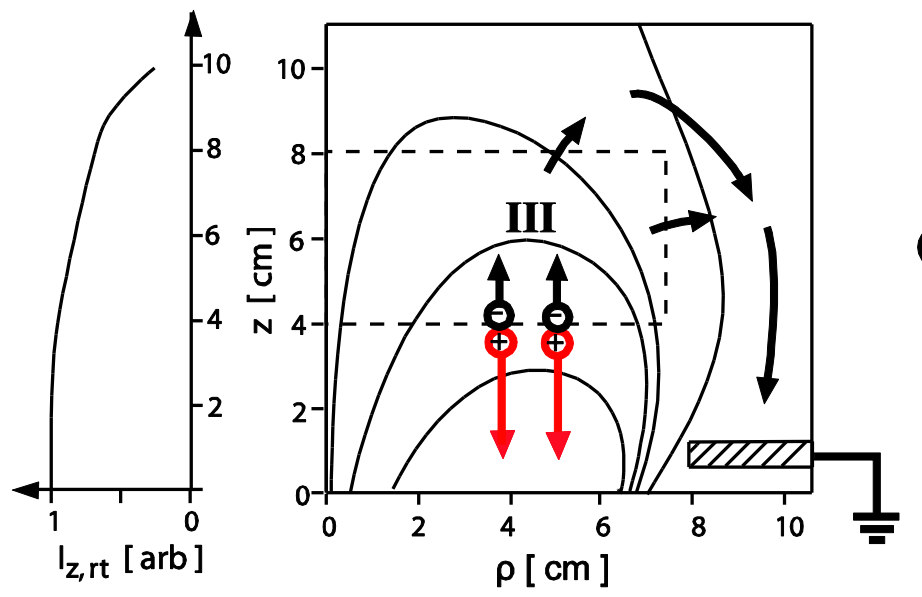

Figure 5. The charged particle transport in three types of current systems in the axial-radial plane schematically drawn in the topology of the magnetron. A dashed-line rectangle encloses the area of measurements in Figure 3. The hatched area to the lower right denotes the grounded anode ring. It is intersected by the "first grounded magnetic field line". The electrons must cross this field line in order to close the electric circuit. (a) Current system of type I, (b) current systems of type IIa-IIc, and (c) current system of type III. The curves to the left in panels (b) and 
(c) show schematically the current in the $z$ direction above the race track for current types IIa-IIc and III; in current system type I, that current is zero for all $z$.

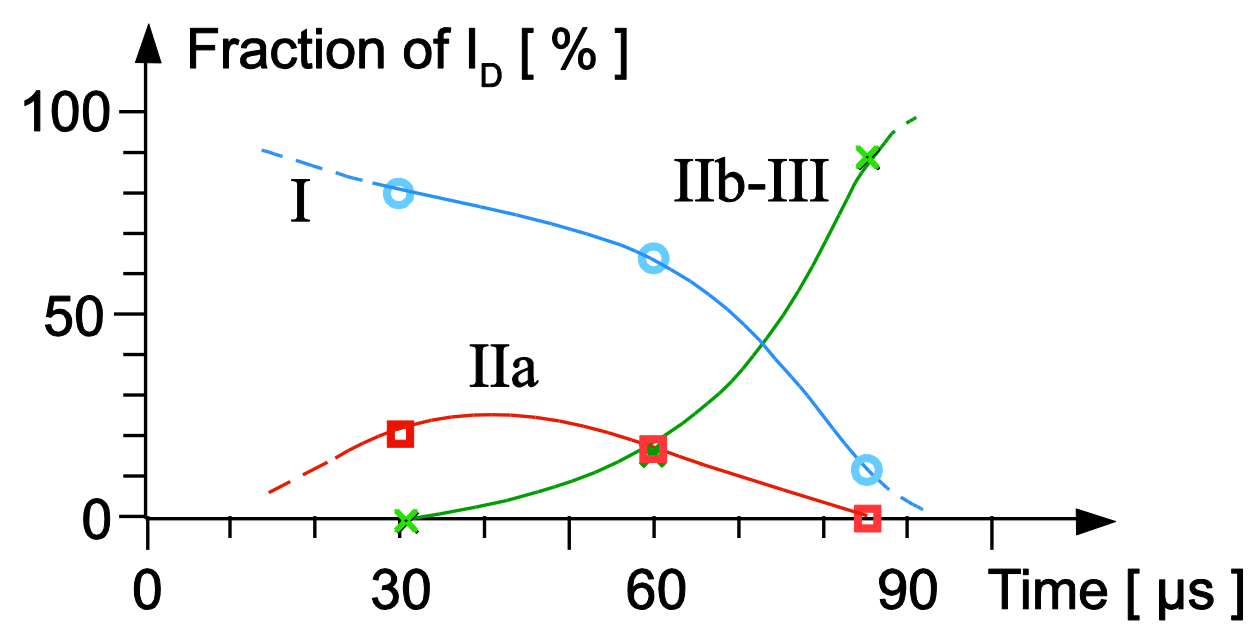

Figure 6. A schematic illustration of the gradual change between current systems of types I, IIa, and the sum of the remaining types IIb-III, as deduced from the data in Figure 4. 


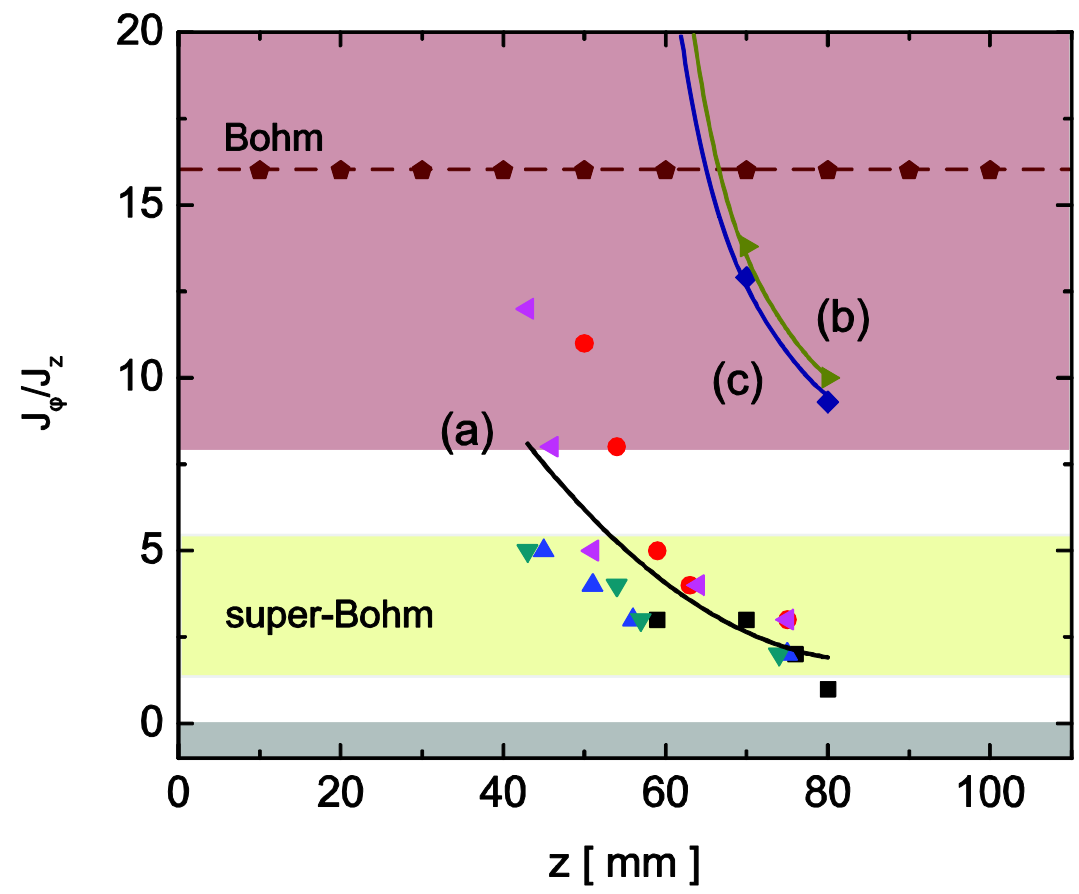

- $30 \mu \mathrm{s}$

- $60 \mu \mathrm{s}$

- $85 \mu \mathrm{s}$

$\nabla \quad 100 \mu \mathrm{s}$

$4 \quad 130 \mu \mathrm{s}$

- Classical coll. (e-n)

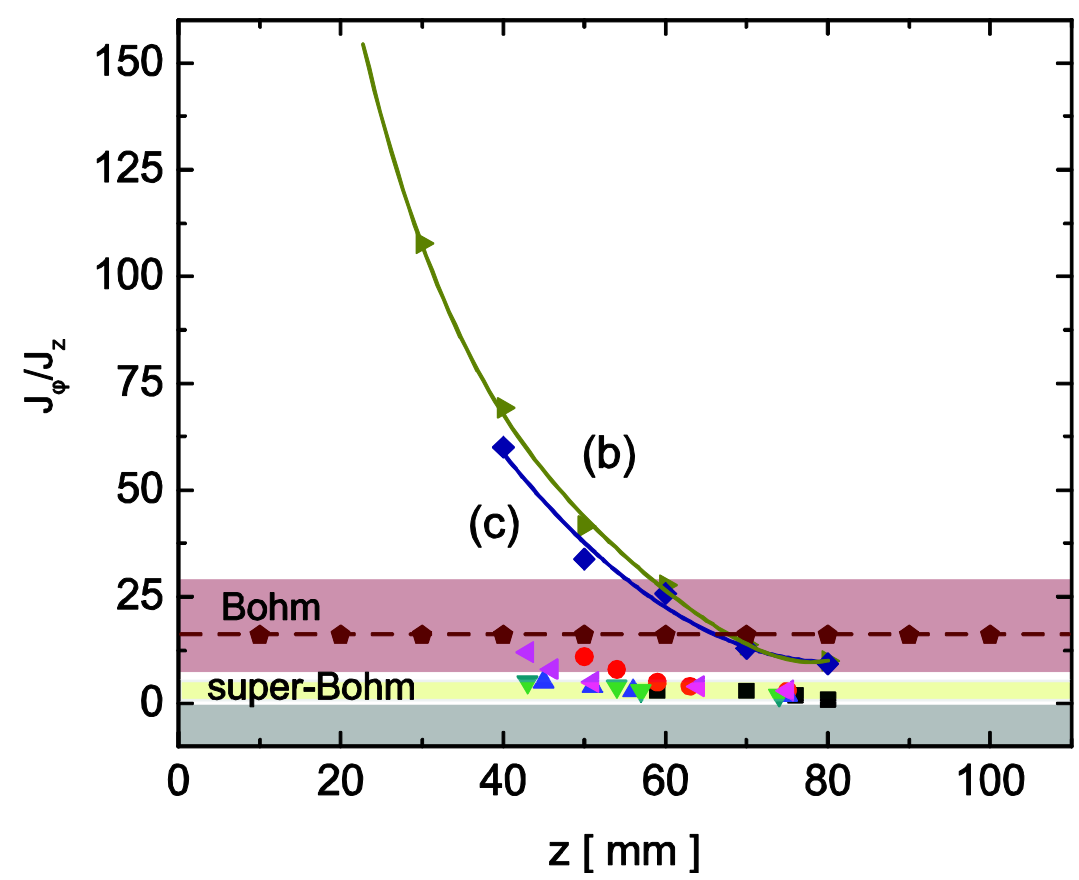

- Classical coll. (e-n, e-i)

- Bohm

Figure 7. Measurements of $J_{\varphi} / J_{z} \approx \omega_{g e} \tau_{E F F}$ above the race track, at the times a - e (Figure 2).

The curve marked (a) is the average of the experimental data. For reference, two values of classical $\omega_{g e} \tau_{\text {class. }}$ are also given: classical electron-neutral collisions (b), and combined electronneutral and Coulomb collisions (c), as well as the Bohm-value $\omega_{g e} \tau_{\text {class. }}=16$ (dashed). The Bohm region, $8<\omega_{g e} \tau_{E F F}<30$, as well as the faster-than-Bohm region (super-Bohm), $1.5<\omega_{g e} \tau_{E F F}<5.5$, appear as shaded areas. The Coulomb collision rate is based on the measurements by 
Sigurjonsson of $T_{e}$ and $n_{e}$ [20]. The bottom panel displays the same data as the top panel, but with an increased range of $J_{\varphi} / J_{z}$. 


\section{References}

[1] Kouznetsov V, Macák K, Schneider J M, Helmersson U and Petrov I 1999 Surf. Coat. Technol. 122290

[2] Helmersson U, Lattemann M, Bohlmark J, Ehiasarian A P and Gudmundsson J T 2006 Thin Solid Films 5131

[3] Bohlmark J, Lattemann M, Gudmundsson J T, Ehiasarian A P, Aranda Gonzalvo Y, Brenning N and Helmersson U 2006 Thin Solid Films 5151522

[4] Lundin D, Larsson P, Wallin E, Lattemann M, Brenning N and Helmersson U 2008 Plasma Sources Sci. Technol. 17035021

[5] Samuelsson M, Lundin D, Jensen J, Raadu M A, Gudmundsson J T and Helmersson U 2010 Surf. Coat. Technol. 15, 591

[6] Ehiasarian A P, Wen J G and Petrov I 2007 J. Appl. Phys. 101054301

[7] Alami J, Persson P O A, Music D, Gudmundsson J T, Bohlmark J and Helmersson U 2005 J. Vac. Sci. Technol. A 23278

[8] Aijaz A, Lundin D, Larsson P and Helmersson U 2010 Surf. Coat. Technol. 204 2165

[9] Wallin E, Selinder T I, Elfwing M and Helmersson U 2008 Europhys. Lett. 82 36002

[10] Lundin D, Helmersson U, Kirkpatrick S, Rohde S and Brenning N 2008 Plasma Sources Sci. Technol. 17025007

[11] Bohlmark J, Helmersson U, VanZeeland M, Axnäs I, Alami J and Brenning N 2004 Plasma Sources Sci. Technol. 13654

[12] Rossnagel S M and Kaufman H R 1987 J. Vac. Sci. Technol. A 52276 
[13] Bradley J W, Thompson S and Aranda Gonzalvo Y 2001 Plasma Sources Sci. Technol. 10490

[14] Brenning N, Axnäs I, Raadu M A, Lundin D and Helmerson U 2008 Plasma Sources Sci. Technol. 17045009

[15] Brenning N, Merlino R L, Lundin D, Raadu M A and Helmersson U 2009 Phys. Rev. Lett. 103225003

[16] Hutchinson I H 2002 Principles of Plasma Diagnostics (2 ${ }^{\text {nd }}$ ed.) (Cambridge) Cambridge University Press p 15-17

[17] Argueso M, Robles G and Sanz J 2005 Rev. Sci. Instrum. 76065107

[18] Bohlmark J, Gudmundsson J T, Alami J, Lattemann M and Helmersson U 2005 IEEE Trans. Plasma Sci. 33346

[19] Chen F F 1984 Introduction to Plasma Physics and Controlled Fusion Vol. I: Plasma Physics (New York) Plenum p 179

[20] Sigurjonsson P 2008 Spatial and temporal variation of the plasma parameters in a high power impulse magnetron sputtering (HiPIMS) discharge (Reykjavik) Faculty of Engineering, University of Iceland 